\title{
Konsequenzen der nationalen E-Health-Strategie
}

\section{Martin Denz*}

* Dr. med. Martin D. Denz, Executive Master in eGovernance EPFL; Präsident Schweizerische Gesellschaft für Telemedizin und

E-Health; Vice-President European Health Telematics Association; Vorstandsmitglied des Vereins eCH für eGovernment-Standards; ehemaliger Leiter der E-HealthAbteilung FMH.
Korrespondenz:

Dr. med. Martin Denz

iHealth

Grubenweg 8

CH-4153 Reinach

\section{Die E-Health-Strategie ist da}

Der Entwurf einer nationalen Strategie «E-Health» wurde dem Bundesrat am 15. Dezember 2006 zur Kenntnis vorgelegt. Die Anhörung dazu dauert bis zum 30. März 2007, interessierte Kreise können Stellung nehmen [1]. Gestützt auf die Anhörungsergebnisse wird das EDI dem Bundesrat im Frühjahr die Strategie zur definitiven Beschlussfassung vorlegen.

Die Strategie beruht auf einer Vision, welche die übergeordnete Projektidee umschreibt. Auch wenn die daraus abgeleiteten Handlungsfelder und Ziele bei der Umsetzung und Weiterentwicklung der Strategie an neue Bedürfnisse und veränderte Rahmenbedingungen angepasst werden können - die Vision dient als langfristige Richtschnur: «Die Menschen in der Schweiz können im Gesundheitswesen den Fachleuten ihrer Wahl unabhängig von Ort und Zeit relevante Informationen über ihre Person zugänglich machen und Leistungen beziehen. Sie sind aktiv an den Entscheidungen in Bezug auf ihr Gesundheitsverhalten und ihre Gesundheitsprobleme beteiligt und stärken damit ihre Gesundheitskompetenzen. Die Informations- und Kommunikationstechnologie wird so eingesetzt, dass die Vernetzung der Akteure im Gesundheitswesen sichergestellt ist und dass die Prozesse qualitativ besser, sicherer und effizienter sind.»

Im Vordergrund steht der Nutzen für das Individuum, sei es als Bürgerin oder als Patient. Die nationale E-Health-Strategie soll dazu beitragen, den Zugang zu einem bezüglich Qualität, Effizienz und Sicherheit hochstehenden und kostengünstigen Gesundheitswesen zu gewährleisten. Die Technologie steht als «Enabler» im Hintergrund. Es geht nicht um «E»-Health, sondern um «Health» unter Einbezug zeitgemässer Arbeitsinstrumente [2].

Es war weder das Ziel noch standen ausreichende Ressourcen zur Verfügung, um eine «allumfassende» Strategie zu erstellen, die als fertiger Plan umgesetzt werden könnte. Letzteres entspräche weder den (gesundheits-)politischen Realitäten und Mechanismen der Schweiz noch würde dies der Dynamik technologischer Entwicklungen gerecht. Die vorliegende E-HealthStrategie schlägt vielmehr Leitplanken für die
Zukunft vor. Es ist wünschenswert, dass darüber eine lebhafte Diskussion in Gang kommt [3].

\section{Was kommt auf uns zu?}

Je intensiver die Schweiz über die E-HealthStrategie diskutiert, umso deutlicher wird zu erkennen sein, dass E-Health einen Bezugspunkt braucht: eine übergeordnete Gesundheitsstrategie für die Schweiz. Wir benötigen eine Klärung darüber, was wir denn überhaupt wollen - die vermeintliche Technologiediskussion ist de facto eine gesundheitspolitische Diskussion! Es geht nicht um E-Health, sondern darum, wie und in welche Richtung sich unser heutiges Gesundheitswesen weiterentwickeln soll [4,5].

Diese Diskussion wird nicht allen angesprochenen Akteuren gefallen. Es wäre doch viel einfacher, weiterhin reaktiv und planlos an unserem Gesundheitssystem «herumzudoktern» [6]. Könnte es sein, dass sich die Akteure auf eine Symptomtherapie des kranken Schweizer Gesundheitswesens spezialisiert haben statt auf kausale Behandlungsstrategien? Könnte es sein, dass niemand ernsthaft möchte, dass sich etwas verändert? Könnte es sein, dass man den Sack (die E-Health-Strategie) statt den Esel (drohende Veränderungen) schlägt? [7]

E-Health ist Ausdruck eines anstehenden Transformationsprozesses. Wie aus anderen Sektoren und Industrien zu lernen ist, können durch den Einsatz von Informations- und Kommunikationstechnologien (ICT) bestehende Prozesse, Strukturen und Dienstleistungen optimiert werden. Durch den Einsatz von ICT entstehen auch neue Prozesse und Dienstleistungen, wünschenswerterweise geplant, nicht selten auch unverhofft (Beispiel: Mobiltelephon). Manches wird durch die ICT überflüssig werden, besonders Strukturen und Prozesse, die allzulange weder auf ihren Sinn noch ihre Bedarfs- und Nutzenorientierung hinterfragt worden sind [8].

\section{Erfahrungen mit E-Health im Ausland}

In denjenigen Ländern, die entweder über eine nationale E-Health Strategie verfügen und daran sind, E-Health auf nationaler Ebene umzusetzen, sind folgende Gemeinsamkeiten zu identifizieren: 
- erfolgreiche nationale E-Health-Aktivitäten sind verknüpft mit dem Vorhandensein von mindestens einem dieser Faktoren: nationale Gesundheitsreform, übergeordnete Gesundheitsstrategie, «Commitment» eines hohen Entscheidungsträgers für E-Health;

- Aufbau von Koordinations- und Kompetenzzentren auf nationaler, regionaler und supranationaler Ebene (Beispiel: EU);

- die Erarbeitung einer nationalen E-HealthArchitektur, d.h. einer Gesamtsicht darüber, wie die Elemente eines elektronisch unterstützten Gesundheitswesens miteinander funktionieren sollen, und der Konsens, was damit erreicht werden soll;

- um dieses übergeordnete Ziel zu erreichen, wird auf ein «Re-Engineering» aller Prozesse des Gesundheitswesens verzichtet und pragmatisch vorgegangen: Man definiert wesentliche Bausteine von E-Health (unter Berücksichtigung jeweiliger nationaler Besonderheiten und Bedürfnisse), also die Elemente, die als Ecksteine einer künftigen E-HealthInfrastruktur dienen werden;

- auf dieser Basis sollen sich neue Strukturen und Prozesse entwickeln - teils bekannten Bedürfnissen im Gesundheitswesen folgend, sich teils an marktwirtschaftlichen Mechanismen ausrichtend. $\mathrm{Zu}$ den oft anzutreffenden E-Health-Bausteinen gehören keineswegs nur technische Elemente, sondern auch Elemente mit normativem und organisatorischem Charakter.

\section{E-Health und Patientensicherheit}

1999 wies das Institute of Medicine (IOM) mit seiner Publikation «To Err is Human: Building a Safer Health System» nach, dass Patienten im Zusammenhang mit medizinischen Behandlungen geschädigt werden [9]. Dabei handelt es sich nicht um «Kunstfehler» im engeren Sinne, sondern um die Kombination von ungenügendem Informations- und Wissensmanagement, menschlichem und organisatorischem Versagen. Es handelt sich um ein weltweites Problem: Bis zehn Prozent aller Behandlungen führen zu vermeidbarer (teils tödlicher) Schädigung der Patienten [10].

Es geht bei der Patientensicherheit nicht nur um die Vermeidung individuellen menschlichen Leids und um die Verbesserung der Organisation und Qualität medizinischer Behandlung, sondern auch um die Vermeidung damit verbundener, erheblicher Folgekosten. Die WHO, der Europarat, viele Regierungen und internationale medizinische Organisationen unternehmen für die «Patient Safety» grosse Anstrengungen, um die
Sicherheit der Patienten innerhalb ihrer Gesundheitssysteme zu verbessern.

In der Schweiz ist das Thema Patientensicherheit nach wie vor tabu - wir sind davon überzeugt, dass unser Gesundheitssystem besser und sicherer ist. In denjenigen Ländern, wo die Patientensicherheit einen prioritären Stellenwert in der gesundheitspolitischen Agenda einnimmt, wurden nicht nur zusätzliche gesetzgeberische Massnahmen ergriffen, sondern gezielt auch E-Health gefördert. Die besondere Förderung von E-Health durch die europäischen Regierungen ist dadurch zu erklären, dass der Einsatz der ICT entscheidend zur Verbesserung der Patientensicherheit beiträgt [11].

Die im Strategieentwurf vorgeschlagenen Handlungsfelder «Elektronisches Patientendossier» und «Onlineinformationen und Onlinedienste» stehen in direktem Zusammenhang mit der Verbesserung der Patientensicherheit. E-Health kann mit folgenden Ansätzen dazu beitragen:

- Identitätsmanagement: Vermeidung von Verwechslungen, eindeutige Zuordnung von Produkten und Behandlungsmassnahmen;

- elektronisches Patientendossier: Verfügbarkeit von Informationen zu Gunsten diagnostischer und therapeutischer Massnahmen, Förderung der Zusammenarbeit der Behandelnden, Koordination und Unterstützung von komplexen und Langzeitverläufen;

- Medikamentenmanagement: Vermeidung von Behandlungsfehlern durch den Einsatz elektronischer Entscheidungsunterstützung (decision support systems), Vermeidung von Dosierungs- und Verschreibungsfehlern durch «computer physician order entry» (CPOE), Langzeitbehandlungs- und Compliancemanagement;

- Evidence-based Medicine: realitätsbasierte Datenerhebung durch Erfassung von «best practice» und ambulantes Telemonitoring;

- «Health literacy»: Förderung von Gesundheitskompetenz und Selbstverantwortung, Zugriff auf persönliche Daten und Nutzung derselben [12];

- Standardisierung und Interoperabilität: bessere Zusammenarbeit und Datenaustausch, Fehlervermeidung und Förderung der Fehlerkultur durch den Einsatz von «critical incident reporting systems»;

- Epidemiologie: statistische Früherfassungsund Warnsysteme.

\section{Das Beispiel Dänemark}

Ein kürzlich veröffentlichter Bericht über eine Studienreise nach Dänemark zeigt positive Ein- 
drücke über das dänische Gesundheitssystem [13]. Die Veränderungen im dänischen Gesundheitssystem sind eng verknüpft mit der Integration der ICT. Am Beispiel von Dänemark kann der Zusammenhang zwischen E-Health und einer Reform des Gesundheitssystems gut illustriert werden [14-16]: Die per 1. Januar 2007 abgeschlossene Reform wurde durch den radikalen Umbau föderalistischer, administrativer und Versorgungsstrukturen möglich. Dänemark bestand bis 1970 aus 24 «counties» (Kantone) und aus über 1300 «municipalities» (Gemeinden). Im Rahmen einer Reform des öffentlichen Sektors erfolgte zuerst eine Reduktion auf 14 Kantone, jetzt wurden sie in fünf landesweite Gesundheitsregionen übergeführt. Die Gemeinden wurden durch Fusionen um den Faktor fünf auf nur noch 275 zusammengefasst. Die Organisations- und Versorgungsstrukturen wurden verschlankt, die Hälfte der Spitalbetten gestrichen.

Gleichzeitig erfolgte eine Neuzuteilung von Aufgaben und Verantwortlichkeiten im Gesundheitswesen. Zu diesem Zweck wurden regionale Strukturen aufgewertet, die fünf landesweiten Gesundheitsregionen durch die Wahl von 41 Regionalvertretern besser auf die Wahrnehmung dezentraler Bedürfnisse ausgerichtet. Auf die Schweiz übertragen würde das bedeuten, dass unsere 26 Kantone auf ein Fünftel reduziert und funktionale Einheiten zu Gunsten des Gesundheitswesens geschaffen werden, die an die militärischen Territorialzonen der Schweiz erinnern!

Die neuen Organisationseinheiten erhielten neue Aufgaben zugeteilt für die medizinische Versorgung und Public-Health-Aufgaben, unterstützt durch neue Finanzierungsmodelle und

Abbildungen 1

E-Health-Architektur.

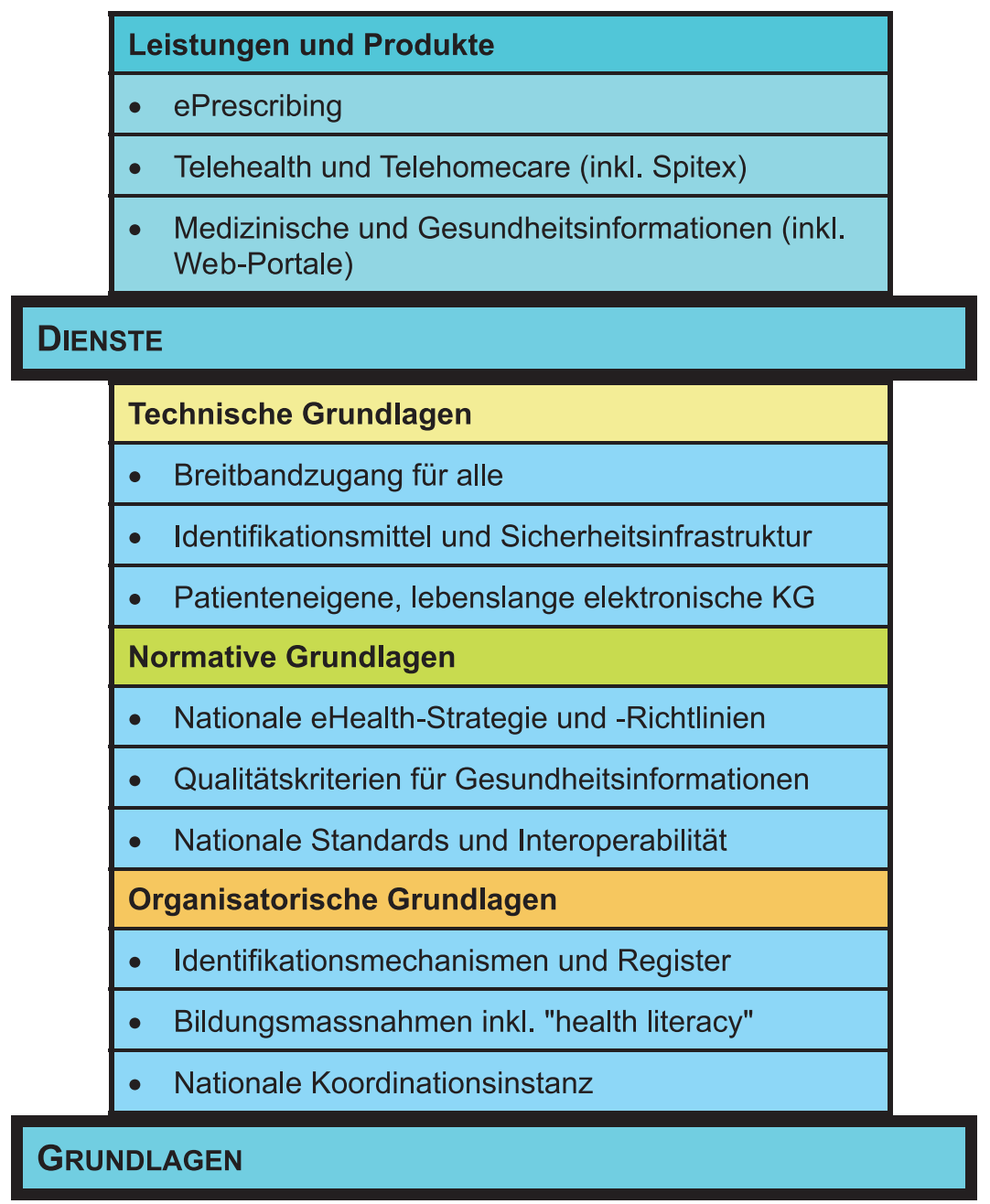


Anreizsysteme. Gleichzeitig erfolgten eine Aufwertung der allgemeinmedizinischen Grundversorger sowie Investitionen in E-Health für den elektronischen Datenaustausch und den Aufbau elektronischer Dienste [17]. Folgende drei Schwerpunkte würden dadurch zu Gunsten der Patienten umgesetzt:

1. kurze und garantierte (!) minimale Wartezeiten;

2. Informationen über den Zugang zum (individuell wählbaren) Leistungserbringer und Spital;

3. Aufbau eines öffentlichen Systems von Qualitätsindikatoren.

Dazu sei erwähnt, dass Dänemark seit Mitte der neunziger Jahre den konsequenten Auf- und Ausbau von «MedCom» (Danish Healthcare Data Network) [18] vorangetrieben hatte, ähnlich einer «nationalen Medidata». Diese PublicPrivate-Partnerschaft entwickelte sich zu einer unersetzlichen Plattform für das dänische Gesundheitswesen, ebenso stellt sie die E-HealthInfrastruktur für weitere nordische Länder bereit. Die seit 1995 gewonnen Erfahrungen und die dabei entwickelten Standards werden für den Auf- und Ausbau der E-Health-Infrastruktur der europäischen Mitgliedstaaten übernommen.

Im Verlaufe der Rationalisierung bestehender Versorgungsstrukturen bekamen die verantwortlichen Gesundheitspolitiker Dänemarks Angst vor ihrem eigenen Mut. Sie befürchteten, dass die dänische Bevölkerung den Eindruck haben könnte, ihr Zugang zu Gesundheitsdienstleistungen werde wegrationiert. Um dem entgegenzusteuern, wurde das Webportal «www. sundhed.dk», das zunächst für den Daten- und Informationsaustausch zwischen Leistungserbringern konzipiert worden war, als Serviceportal zu Gunsten der Bevölkerung erweitert. Daraus entwickelte sich eine alle Erwartungen übertreffende Nachfrage. Die Bevölkerung nimmt das nationale Gesundheitsportal nicht als «Kompensation» einer rationalisierten Gesundheitsversorgung wahr, sondern wird aufgrund seines praktischen Nutzens (Beispiel: webbasierte Termineinschreibung beim Hausarzt) und Mehrwerts intensiv genutzt und geschätzt [19].

\section{Schlussfolgerungen}

E-Health stellt die Instrumente für eine wissensbasierte Leistungserbringung und für gesundheitspolitisch notwendige Entscheidungsdaten bereit. E-Health wird zur Steuerung der Prozesse beitragen und die Diskussionen auf Prozess- und Dienstleistungsinnovation hinführen [20]. Die auch bei uns vorhandenen Probleme in Sachen
Patientensicherheit können dank E-Health zugleich erkannt und verbessert werden.

Mit einer E-Health-Strategie alleine sind aber keine Probleme zu lösen. Wir brauchen eine übergeordnete nationale Gesundheitsstrategie. Die Verantwortungsträger sollten weniger Aufgabenverzichtsplanung und mehr Innovationsförderung im Gesundheitswesen betreiben. Bund und Kantone müssen einen expliziten Budgetanteil ihrer Gesundheits- und Präventionsaktivitäten für E-Health vorsehen sowie Anreize für Investitionen in die E-Health-Infrastruktur setzen [21].

Jede Strukturveränderung löst verständlicherweise Ängste und Widerstände aus. Besonders in der Schweiz, wo wir uns darauf fokussieren, was wir verlieren könnten. Die Schweiz hat aber auch Zeiten der Aufbruchstimmung erlebt, in denen wir fähig waren, wahrzunehmen, was wir durch Veränderungswillen und Innovationskraft hinzugewinnen könnten. Auf dieser Geisteshaltung beruhen nicht nur wirtschaftliche Erfolge, sondern auch die Fortschritte in der Medizin [22].

E-Health bedeutet eine medizinische, gesellschaftliche und volkswirtschaftliche Chance. Um diese Chance wahrzunehmen, muss mehr gegenseitiges Vertrauen und Glaubwürdigkeit zwischen den Akteuren geschaffen werden. Solange wir uns darauf konzentrieren, was wir verlieren könnten, statt darauf, was wir gewinnen könnten, wird sich nichts verbessern - ob mit oder ohne E-Health. Alle Akteure im Schweizer Gesundheitswesen hätten mit E-Health etwas zu gewinnen - wer wagt, gewinnt!

1 www.bag.admin.ch/themen/krankenversicherung/ 00305/03505/index.html?lang=de. [Website 18.02.07]

2 Denz M. Neue Technologien: mit E-health von High-tech zu High-touch. Schweiz Ärztezeitung. 2001;82(42):2251-6.

3 Denz M. Plädoyer für eine nationale E-HealthStrategie. E-Health als musikalisches Gesamtkonzept. Schweiz Ärztezeitung. 2005;86(7):407-9.

4 Public Health Schweiz. Gesundheitsziele für die Schweiz. http://www.public-health.ch/site/ 24.0.html [Website 18.02.07]

5 Ministry of Social Affairs and Health Finland. Health in All Policies: Prospects and potentials. European Observatory on Health Systems and Policies, 2006.

6 Sommer J (Hrsg). Muddling Through Elegantly: Rationierung im Gesundheitswesen. Basel: EMH Schweizerischer Ärzteverlag. 2001.

7 OECD. OECD-Berichte über Gesundheitssysteme: Schweiz. Genf: OECD, WHO; 2006.

8 Porter M, Olmsted Teisberg E. Redefining Health Care: Creating Value-Based Competition on Results. Harvard Business School Press. 2006 
9 Kohn L, Corrigan J, Donaldson M (eds.). To Err Is Human: Building a Safer Health System. National Academies Press. 2000.

10 HARM study (final report). Hospital Admission Related to Medication. Division of Pharmacoepide miology \& Pharmacotherapy, Utrecht Institute for Pharmaceutical Sciences. The Netherlands. November 2006

\section{Telematiktage Bern 2007}

Healthcare Forum, Donnerstag, 8. März, Bern

Die Telematiktage Bern stellen mit dem diesjährigen Programm die Erfolgsfaktoren und zukunftsgerichteten Strukturen dar, die zur Umsetzung von E-Health in der Schweiz beitragen.

Folgende Fragen sollen beantwortet werden: Wo steht die Umsetzung von E-Health in der Schweiz heute? Welche regionalen Lösungsansätze haben sich diesseits und jenseits der Landesgrenze bewährt? Wo gibt es Gemeinsamkeiten, und wo liegen die Unterschiede? Welches sind die bestimmenden Erfolgsfaktoren? Wo liegen die Hürden in der Umsetzung?

Regionale E-Health-Projekte - Lösungsansätze und Erfolgsfaktoren

Die heutige Situation im Schweizer Gesundheitswesen ist gekennzeichnet durch unzureichende Steuerung, mangelnde Vernetzung zwischen Leistungserbringern und Kostenträgern, fehlende Normen und Standards sowie isolierte, nicht aufeinander abgestimmte Anwendungen. Sollen Effektivität und Effizienz der medizinischen Leistungsprozesse gesteigert werden, müssen die heute bestehenden Insellösungen mittels neuer Organisationsformen, Technologien und Prozesse verbunden werden.

Vernetzt, statt isoliert agieren

Regionale integrierte Gesundheitsnetze sind der grosse Hoffnungsträger im Gesundheitswesen. Als Prämisse für künftige Prozessinnovationen sollen sie Haus- und Fachärzte, Spitäler, Apotheken und Labors, aber auch Pflegeangebote, Rehabilitationskliniken, Versicherer und Patienten optimal miteinander vernetzen - zum Nutzen und zum Wohl der Patientinnen und Patienten. Heute sind in verschiedenen Regionen Netzwerke im Aufbau, und aus dem Ausland liegen bereits erste konkrete Erfahrungen vor.

Weitere Informationen: www.telematiktage.ch
11 Commission of the European Communities, Information Society \& Media DG. Connected Health: Quality and Safety for European Citizens. Report of the Unit ICT for Health in collaboration with the i2010 sub-group on eHealth and the eHealth stakeholders' group. Brussels. 2006.

12 Wang J, Schmid M. Swiss Health Literacy Survey: Initial findings 2006. www.futurepatient.ch [Website 18.02.07]

13 Zürcher H, Metzger K. Positive Eindrücke vom dänischen Gesundheitssystem. Schweiz Ärztezeitung. 2007;88(3):106-8.

14 Appel M. Public sector reform - a bill has been proposed. Health Policy Monitor, March 2005. http://www.hpm.org/survey/dk/a5/4 [Website 18.02.07]

15 Appel M. A new Health Care Law. Health Policy Monitor, March 2005. http://www.hpm.org/ survey/dk/a5/2 [Website 18.02.07]

16 Pedersen K. Implementation of health care reform. Health Policy Monitor, November 2006. http://www.hpm.org/survey/dk/a8/6 [Website 18.02.07]

17 National Board of Health. National IT Strategy 2003-2007 for the Danish Health Care Service. The Danish Ministry of the Interior and Health. 2003.

18 MedCom. www.medcom.dk/wm109991. [Website 18.02.07]

19 The Danish eHealth Portal. http://www.sundhed.dk/wps/portal/_s.155/ 1912? ARTIKELGRUPPE_ID_=1023050919180045 [Website 18.02.07]

20 economiesuisse. E-Health: Schlüssel zu Prozessinnovation und Qualitätsverbesserung im Gesundheitswesen. Positionspapier, 16.1.2007.

21 Denz M. Integrierter Einsatz von E-Health. digma Zeitschrift für Datenrecht und Informationssicherheit. 2006;(4):160-3.

22 Michel-Alder E, Berchtold P (Hrsg.). Die Umarmung des Hippokrates: Systemintegration im Gesundheitswesen. EMH Schweizerischer Ärzteverlag, Basel, 2003. 